Journal of Computer Science 8 (8): 1329-1337, 2012

ISSN 1549-3636

(C) 2012 Science Publications

\title{
An Enhanced Approach for Medical Brain Image Enhancement
}

\author{
Umamaheswari, J. and G. Radhamani \\ Department of Computer Science, School of Information Technology and Science, \\ GRD College of Science, Affiliated to Bharathiyar University, Tamilnadu, India
}

\begin{abstract}
Problem statement: One of the most common degradations in medical images is their poor contrast quality and noise. The DICOM image consists of speckle (multiplicative noise). While the image is enhanced, the multiplicative noise present in the image is also enhanced. Approach: This study describes the hybrid method to improve the image quality of Digital Imaging and Communications in Medicine (DICOM) images. The idea of image enhancement technique is to improve the quality of an image for early diagnosis. Then followed by a noise reduction using speckle reduction anisotropic filter. This suggests the use of contrast enhancement methods as an attempt to modify the intensity distribution of the image and to reduce the multiplicative noise. Results: In this research study, a new approach for DICOM image is done by applying contrast stretching and anisotropic diffusion where denoising of multiplicative noise is carried out and the level of contrast is improved. The quality of the image is enhanced and noise free for DICOM image analysis. The effectiveness of hybrid method is proved by quantitative approach. Conclusion and Recommendation: The performance of the proposed study is compared with the existing traditional algorithm and real time medical diagnosis image.
\end{abstract}

Key words: Contrast stretching and histogram equalization, degradation, image enhancement, speckle (multiplicative noise), anisotropic diffusion

\section{INTRODUCTION}

Medical image enhancement technologies have attracted much attention during the diagnosis process. Enhanced medical images are desired by a surgeon to help diagnosis and interpretation because medical image qualities are often deteriorated by artifacts. Nowadays, Medical imaging is the best techniques for monitoring the person's diagnosis process. Most of the diseases are diagnosed by doctors using medical imaging methods. One problem that physician encounter is because of the low quality of medical image. This low quality causes difficulty during the diagnosis. So it is necessary to improve the quality of the medical image. There are so many methods for medical image enhancement to make a better perception Lavania et al. (2011), Nakayama et al. (2009), Kabir et al. (2010) and Al-Samaraie and Saiyd (2008). Because of the simplicity and better efficiency, histogram based algorithms Larrabide et al. (2003), are widely used for contrast enhancement of images.

Image enhancement: Image enhancement operation improves the qualities of an image. They improve an image's contrast and brightness characteristics, reduce its noise content or sharpen its details. Image Corresponding Author: Umamaheswari, J., Department of Computer Science, School of Information Technology and Science, GRD College of Science, Affiliated to Bharathiyar University, Tamilnadu, India enhancement techniques may be grouped as either subjective enhancement or objective enhancement. The subjective enhancement technique may be repeatedly applied in various forms until the observer thinks that the image yields the detail necessary for a particular application. Objective image enhancement corrects an image for known degradations. Here distortions are known and enhancement is not applied arbitrarily. This enhancement is not repeatedly applied but applied once based on the measurements taken from the system.

Image enhancement fall into two broad categories as below:

- Frequency domain technique

Spatial domain refers to the image plane itself and approaches in this categories are based on direct manipulation of pixels in an image. Frequency domain processing techniques are based on modifying the Fourier transform of an image. Spatial domain refers to the total of pixels comprising an image. They operate directly on these pixels. Spatial domain processes will be denoted by the expression:

$$
\mathrm{g}(\mathrm{x}, \mathrm{y})=\mathrm{T}(\mathrm{F}(\mathrm{x}, \mathrm{y}))
$$

- Spatial domain technique 


\section{J. Computer Sci., 8 (8): 1329-1337, 2012}

where,

$\mathrm{f}(\mathrm{x}, \mathrm{y})$ is the input image

$\mathrm{g}(\mathrm{x}, \mathrm{y})$ is the processed image

$\mathrm{T}$ is the function of $\mathrm{F}$

There are four steps involved in applying image enhancement process:

- The first step is image capturing of DICOM Human Brain

- Then, save the images under .jpg extension

- The third step is to select picture with three different type which is normal image, bright image and dark image. Three images are selected for each different type

- $\quad$ The last step is applying the proposed techniques to the selected images

There are so many methods for medical image enhancement to make a better perception from medical images. Various methods have been suggested for increasing medical image's quality in recently years. Contrast enhancement and histogram processing is one of the most important digital image processing techniques and it is widely used for increasing medical image's quality. Because the simplicity and better efficiency of the histogram based algorithms, these algorithms are widely used for contrast enhancement of images. Also it should be mentioned that histogram based techniques are much less expensive when compare to the other methods by Sapiro et al. (1994) Ziaei et al. (2008); Jagatheeswari et al. (2009); Kachouie (2008); Zhao et al. (2010), Ramyashree et al. (2010); Vij and Singh (2011); Mahmoud and Marshal (2008). Studies of frequency domain transform mainly concentrate on the speckle reduction and histogram equalization is a moderately typical method of image enhancement in the spatial field. The Non linear anisotropic diffusion model has been successfully applied in the image processing domain by Perona and Malik (1990) and Gerig et al. (2002).

Speckle noise filtering: Medical images are very difficult to diagnose because of the existence of speckle which hampers the perception and the extraction of fine details from the image. Only skilled radiologists can make an effective diagnosis and hence limiting its use in a wide medical network. For anisotropic diffusion filtering the direction and strength of the diffusion is controlled by an edge detection function. It removes speckles and at the same time enhances the edges. It removes speckles by modifying the image via solving a Partial Differential Equation (PDE). Perona and malik proposed the nonlinear PDE for smoothing image in continual domain.
Different methods have been investigated for speckle reduction. These methods include early methods such as Lee filter, Frost filter, Kuan filter and recently developed methods such as adaptive filters, wavelet transform, bilateral filters, nonlocal- means and anisotropic diffusion filters. An Adaptive Weighted Median Filter (AWMF) for speckle reduction is proposed. Different from the common median filter, AWMF adjusts weight coefficients and smoothing characteristics based on the local statistics.

The methods of image enhancement includes, histogram equalization (2008), adaptive histogram equalization 2011. A method of differential histogram equalization in (2009). At present, there are many types of image denoising and enhancement algorithms, but all these methods are very rarely applied for medical image directly. Removing noise followed by enhancement often make the edges not visible. Enhancement followed the denoising affect is done for medical image. The lot of papers discussed the medical image segmentation in gives Ahmed and Nordin (2011) the idea why one want to improve the quality of medical images.

\section{MATERIALS AND METHODS}

One problem that physician encounter with medical image is low quality of the medical image. To overcome this drawback of image contrast enhancement and denoising methods, anisotropic diffusion method introduced by Perona and Malik (1990) is used. Contrast enhancement techniques are widely used for enhancing visual quality of low contrast images. Histogram Equalization (HE) by contrast stretching is a very popular technique for enhancing the contrast of an image. Its basic idea lies in mapping the gray levels based on the probability distribution of these inputs gray levels. It flattens and stretches the dynamic range of the image's histogram, resulting in overall contrast improvement. $\mathrm{HE}$ is the most prominent technique applied to medical imaging has been applied in various fields such as medical image processing.

Anisotropic diffusion considers a conduction term that is locally computed and depends on the differential structure of the image. Anisotropic diffusion filter was used by Gerig et al. (2002) to enhance MR images. This study describes about the techniques efficiently for MRI images and for CT images through contrast enhancement. The framework for the hybrid approach of is shown in Fig. 1.

Image Enhancement Model (Contrast Stretching): Contrast stretching (often called normalization) is a simple image enhancement technique that attempts to improve the contrast in an image by 'stretching' the range of intensity values which contain a desired range of values. 
J. Computer Sci., 8 (8): 1329-1337, 2012

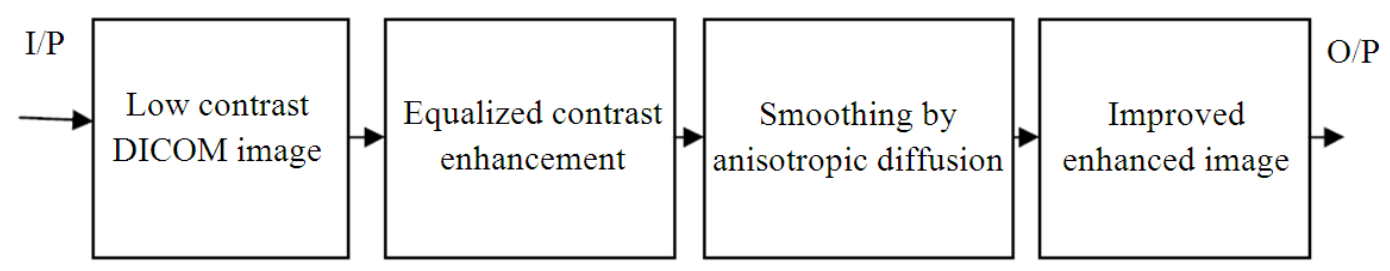

Fig. 1: Proposed approach for image enhancement

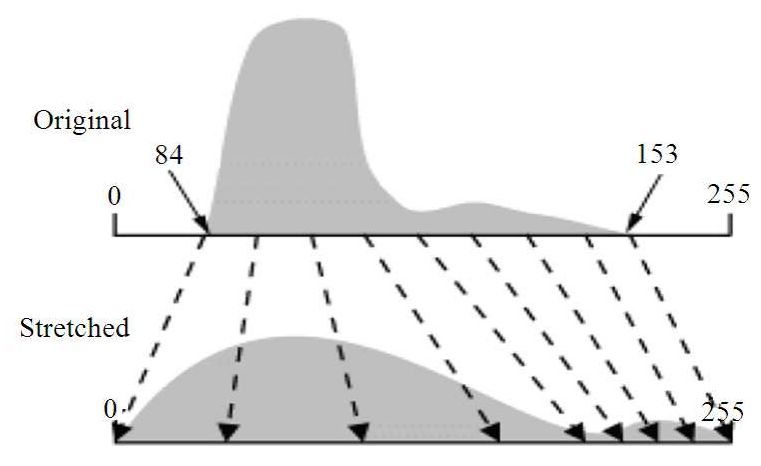

Fig. 2: Equalized contrast stretch

Low contrast occurs due to the poor illumination, while capturing images. The idea of contrast stretching is to increase the dynamic range of intensity level in the processed image. The functioning of the contrast stretching operation on gray scale image is to apply the following equation on each of the pixels in the input image to form the corresponding output image pixel by Ismail and $\operatorname{Sim}(2011)$.

$\mathrm{I}(\mathrm{x}, \mathrm{y})=(\mathrm{I}(\mathrm{x}, \mathrm{y})-\mathrm{min})$

$\left(\frac{\text { No.of.int.Lvel. }}{\max -\min }\right)+$ Inital.Int.Lvel.

where, I ( $x, y)$ represents the images in Eq. (1) and the equation describes about "min" and "max" which is the minimum intensity value and the maximum intensity value in the current image. Normally in the gray level images the lowest possible intensity is 0 and the highest intensity is 255 . The intensity value is stretched to the maximum of 255 which converts the image to be blurred. This occurs when and equalized contrast stretch is applied.

Equalized contrast stretch: This stretch assigns more display values (range) to the frequently occurring portions of the histogram. By this method, the detail in these areas will be better enhanced relative to those areas of the original histogram where values occur less frequently.
Histogram equalization is one of the most useful forms of nonlinear contrast enhancement. When an image's histogram is equalized, all pixel values of the images are redistributed so there are approximately an equal number of pixels to each of the user-specified output gray-scale classes (e.g., 32, 64 and 256).Contrast is increased at the most populated range of brightness values of the histogram (or "peaks"). It automatically reduces the contrast in very light or dark parts of the image associated with the tails of a normally distributed histogram. Histogram equalization can also separate pixels into distinct groups, if there are few output values over a wide range. Histogram equalization is effective only when the original image has poor contrast to start with, otherwise histogram equalization may degrade the image quality.

The following Fig. 2 describes the equalized contrast stretching. Where, the disadvantage of equalized contrast stretch can be overcome by applying linear contrast stretch method. When the histogram is stretched, the range of resultant quality of the image is still not clear. To improve the quality of the image further the linear contrast stretching is processed.

Linear contrast stretch: When the distribution of a histogram in an image is bi or trimodal, an analyst may stretch certain values of the histogram for increased enhancement in selected areas. This method of contrast enhancement is called a piecewise linear contrast stretch. The brightness ranges in the modes of the histogram involves the identification of a number of linear enhancement.

In the normal linear contrast stretch example, the minimum and maximum values are stretched to the values of 0 and 255 at a constant level of intensity. In the piecewise linear contrast stretch, several breakpoints are defined that increase or decrease the contrast of the image for a given range of values. The higher the slope, the narrower the range of values being input from the x-axis. This result in a wider output spread for those same values and thus, increases the contrast for that range of values. A low sloping line results in a lower contrast for the same range of values. Notice the series of linear steps in each histogram that stretches intervals of data at different levels of intensities. 


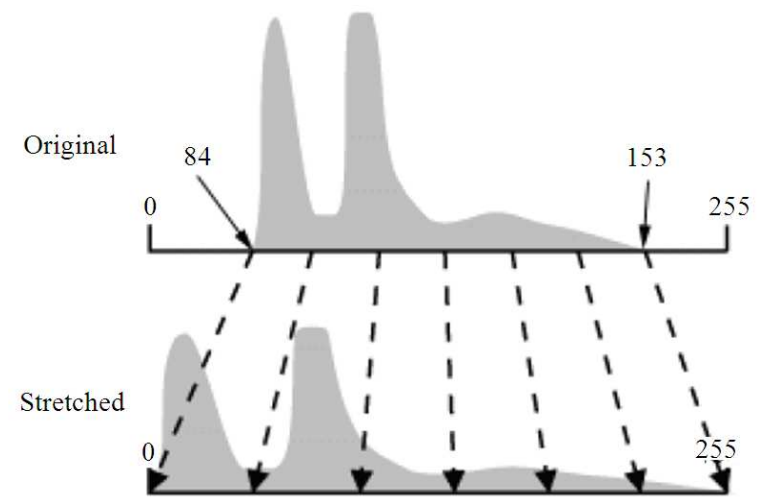

Fig. 3: Linear contrast stretch

This involves identifying lower and upper bounds from the histogram (usually the minimum and maximum brightness values in the image) and applying a transformation to expand the range to the present image size. DN is the input image values from 0 to 255 respectively in the output image and stretching all intervening digital numbers accordingly in Eq. (2).

$\mathrm{DN}_{\mathrm{st}}=255 \mathrm{X} \frac{\left(\mathrm{DN}-\mathrm{DN}_{\min }\right)}{\left(\mathrm{DN}_{\max }-\mathrm{DN}_{\min }\right)}$

where,

$\mathrm{DN}=$ Input Value

$\mathrm{DN}_{\mathrm{st}}=$ Stretching output

The above Fig. 3 shows the linear contrast stretch for an image (Vij and Singh, 2011). Here, the difference among the original and stretched image can be viewed visibly. In original image the histogram starts from the range of 84-255. The stretched image shows the improved enhancement of the image.

Anisotropic diffusion (Speckle noise reduction): During enhancement process of contrast stretching the multiplicative noise present in the image also raises and degrades the image quality. So to suppress the noise anisotropic diffusion model is used. Here Perona Malik (PM) model is used. This model is a partial differential equation based on nonlinear diffusion. The diffusion is similar to the propagation of high concentration regions to low regions in the uneven medium. Two-dimensional nonlinear diffusion equation is shown in formula (3).

$\frac{\partial \mathrm{I}(\mathrm{x}, \mathrm{y}, \mathrm{t})}{\partial \mathrm{t}}=\operatorname{div}(\nabla \mathrm{I})=\frac{\partial^{2} \mathrm{I}}{\partial \mathrm{x}^{2}}+\frac{\partial^{2} \mathrm{I}}{\partial \mathrm{y}^{2}}$

$\mathrm{I}(\mathrm{x}, \mathrm{y}, \mathrm{t})$ is the evolutional matrix of image, $\mathrm{DN}_{\mathrm{st}}$ is the gradient of equalized stretched Image, $\operatorname{div}\left(\mathrm{DN}_{\mathrm{st}}\right)$ is the gradient divergence. In order to denoise and synchronize for protecting the edges, PM model introduces a coefficient transmission of the constraint function. In the flat regions, the speed of processing image transformation will increase. At the edge of the image, the process will decrease automatically. PM model of partial differential equation is constructed in Eq. (4) and (5) as as follow:

$$
\begin{aligned}
& \frac{\partial \mathrm{I}(\mathrm{x}, \mathrm{y}, \mathrm{t})}{\partial \mathrm{t}}=\operatorname{div}[\mathrm{g}(|\nabla \mathrm{I}|) \nabla \mathrm{I}] \\
& \mathrm{g}(\mathrm{r})=\frac{1}{1+\left(\mathrm{r} / \mathrm{k}^{\mathrm{p}}\right)}, \mathrm{p}=1,2
\end{aligned}
$$

In formula (5), $\mathrm{g}(\mathrm{r})$ is a monotonic declined function and the parameter is the value of gradient, so smoothing is able to combine with enhanced output at the same time.

Improved enhancement process: In the Improved Enhancement method, the integrating of linear contrast stretching and anisotropic diffusion is used. The following steps are carried out:

- The low contrast medical image is taken as input

- For the low contrast input, linear contrast stretching is applied because the equalize contrast stretching method does not give clear detail for medical usage

- During enhancement the multiplicative noise present in the medical image also enhanced

- So, to suppress the multiplicative noise the anisotropic diffusion model proposed by Perona Malik is used

- The improved enhancement results with better quality is obtained. The hybrid model is obtain from Eq. 2-4,

$\frac{\partial \mathrm{I}(\mathrm{x}, \mathrm{y}, \mathrm{t})}{\partial \mathrm{t}}=\operatorname{div}\left[\mathrm{g}\left(\left|\mathrm{DN}_{\mathrm{st}}\right|\right) \mathrm{DN}_{\mathrm{st}}\right]$

where, $\mathrm{DN}_{\mathrm{st}}$ is the linear stretching output. It is given as input for the PM model for denoising multiplicative noise to give an improved results. The hybrid model gives better results when compare with traditional enhancement model.

\section{RESULTS}

Experimental results: The DICOM medical images are taken as test images for evaluating results. 
Table 1: Different parametric Evaluation for Image Enhancement algorithms

\begin{tabular}{lrrrlll}
\hline Method & PSNR & \multicolumn{1}{l}{ MSE } & \multicolumn{1}{l}{ RMSE } & UQI & MIE & ET \\
\hline HE & 4.56100 & 22928.74000 & 151.42240 & 0.16580 & 2.52817 & 0.900495 \\
AHE & 19.89849 & 670.85920 & 25.90095 & 0.36298 & 6.07391 & 1.178253 \\
CS & 17.25450 & 1025.23000 & 51.54000 & 0.19540 & 5.24700 & 0.954100 \\
AD & 28.39302 & 57.55295 & 8.89586 & 0.96542 & 6.21865 & 0.697599 \\
HM & 31.34977 & 48.02893 & 6.93020 & 0.97610 & 7.18303 & 0.521550 \\
\hline
\end{tabular}

Where,

$\mathrm{HE}=$ Histogram Equalization

$\mathrm{AHE}=$ Adaptive Histogram Equalization

$\mathrm{CS}=$ Contrast Stretching

$\mathrm{AD}=$ Anisotropic Diffusion

$\mathrm{HM}=$ Hybrid Method

The algorithm is tested in MATLAB. The reconstruction of an image has the dimensions of 256 pixel intensity. The DICOM images contain a wide variety of subject matters and textures. Most of the images used are brain images with normal and abnormal images. This study proposes a hybrid approach to improve the image quality of DICOM images of the early diagnosis process. For enhancing the image contrast stretching is used and further improvement is done by anisotropic diffusion process to suppress the multiplicative noise. The following Table 1 shows the different parametric evaluation for image enhancement algorithms.

Peak signal to noise ratio: The PSNR is defined in logarithmic scale, in db (decibels). The Table 1 shows the parametric evaluation for mixed noise removal. The image metric PSNR is defined as Eq. 6 and 7:

$$
\mathrm{PSNR}=20 \log _{10}\left(\frac{\mathrm{MAX}_{\mathrm{I}}}{\sqrt{\mathrm{MSE}}}\right)
$$

Mean Square Error (MSE): The metric MSE is defined as Eq. 8:

$$
\operatorname{MSE}=\frac{1}{m n} \sum_{i=0}^{m-1} \sum_{j=0}^{n-1}\|I(i, j)-K(i, j)\|^{2}
$$

For two $\mathrm{m} \times \mathrm{n}$ monochrome images $\mathrm{I}$ and $\mathrm{K}$, one image is considered a noisy approximation of the other.

Root-Mean-Square Error (RMSE): is a frequently used measure of the differences between values predicted by a model or an estimator and the values actually observed from the models are estimated Eq. 9:

$$
\operatorname{RMSE}(\mathrm{x})=\sqrt{\operatorname{MSE}(\mathrm{x})}
$$

The Fig. 6 shows the PSNR for image enhancement algorithms.

Universal Quality Index (UQI): It measures image similarity across distortion types. Distortions in UQI are measured as a combination of three factors; Loss of correlation, Luminance distortion and Contrast distortion. Let $\left\{\mathrm{x}_{\mathrm{i}}\right\}$ and $\left\{\mathrm{y}_{\mathrm{i}}\right\}=1,2, \ldots, \mathrm{N}$ be the original and the test image signals, respectively. The universal quality index is Eq. 10 and 11:

$$
\mathrm{UQI}=\frac{4 \sigma_{\mathrm{xy}} \overline{\mathrm{xy}}}{\left[\sigma_{\mathrm{x}}^{2}+\sigma_{\mathrm{x}}^{2}\right]\left[(\overline{\mathrm{x}})^{2}+(\overline{\mathrm{y}})^{2}\right]}
$$

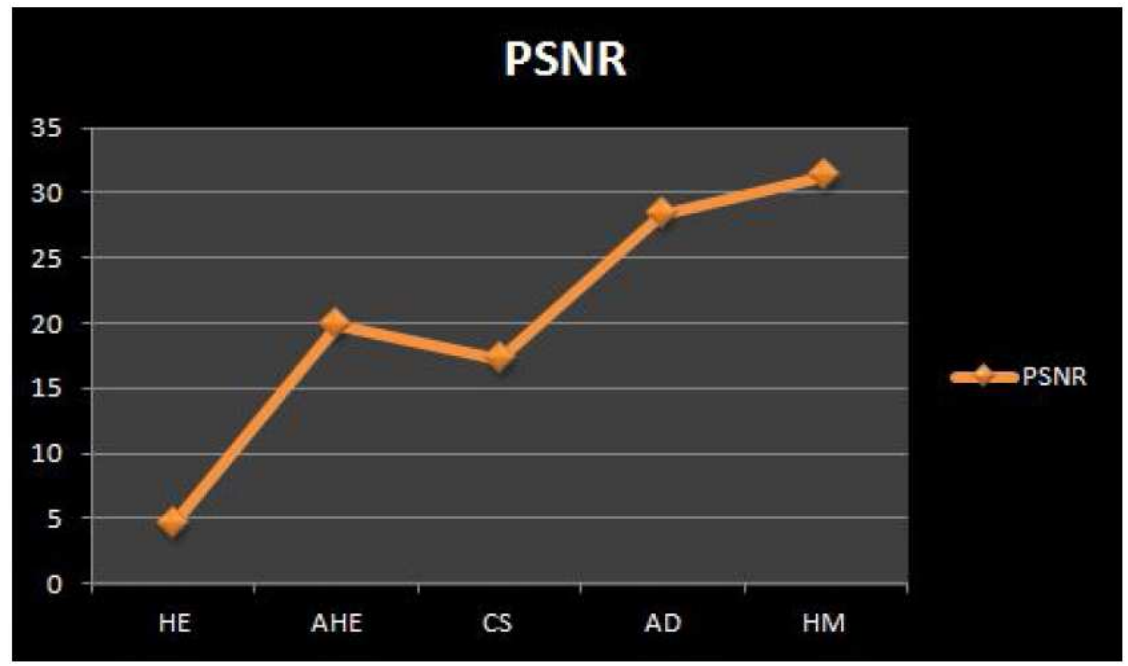

Fig. 4: PSNR results for image enhancement algorithms 


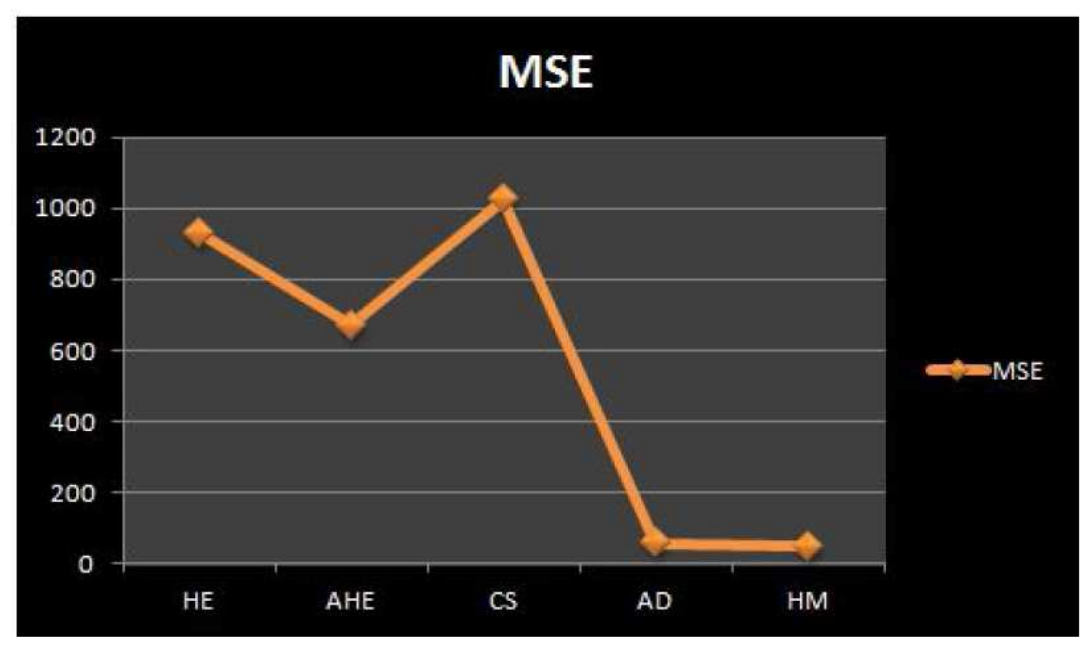

Fig. 5: MSE results for image enhancement algorithms

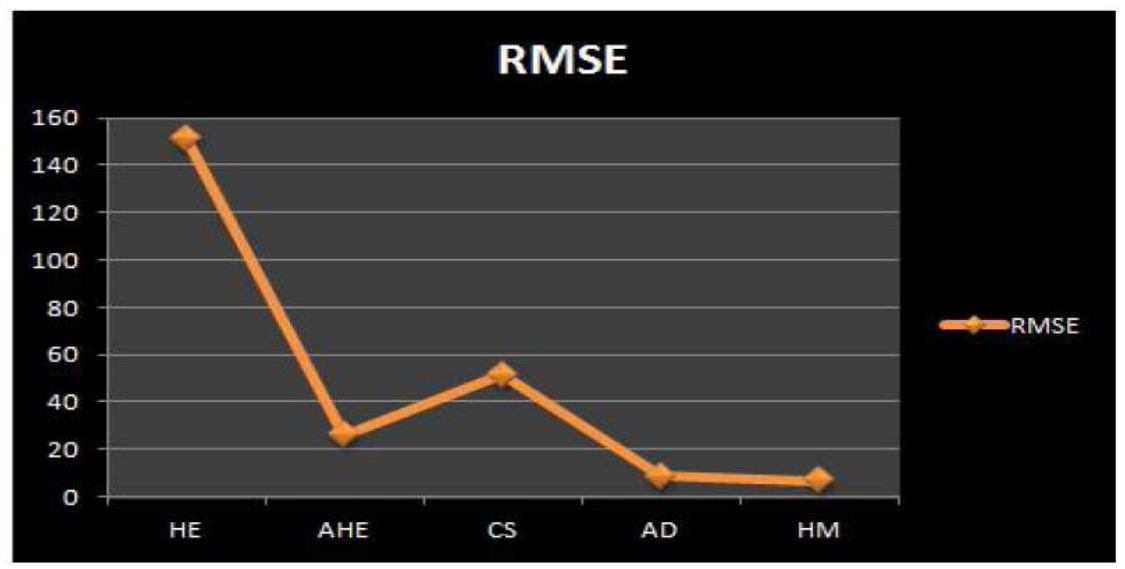

Fig. 6: RMSE results for image enhancement algorithms

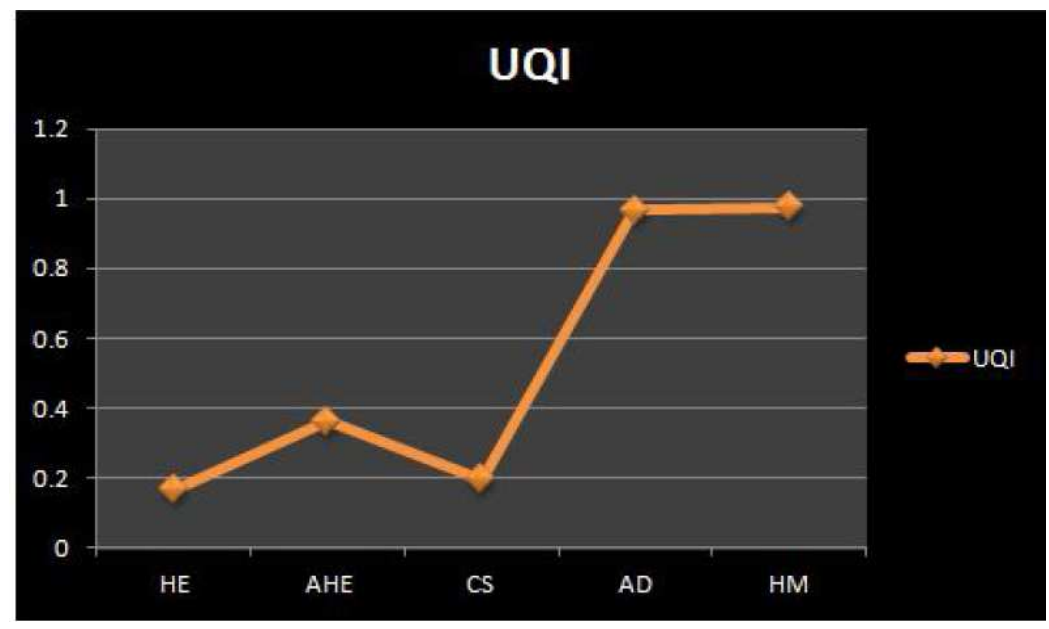

Fig. 7: UQI results for image enhancement algorithms 1334 


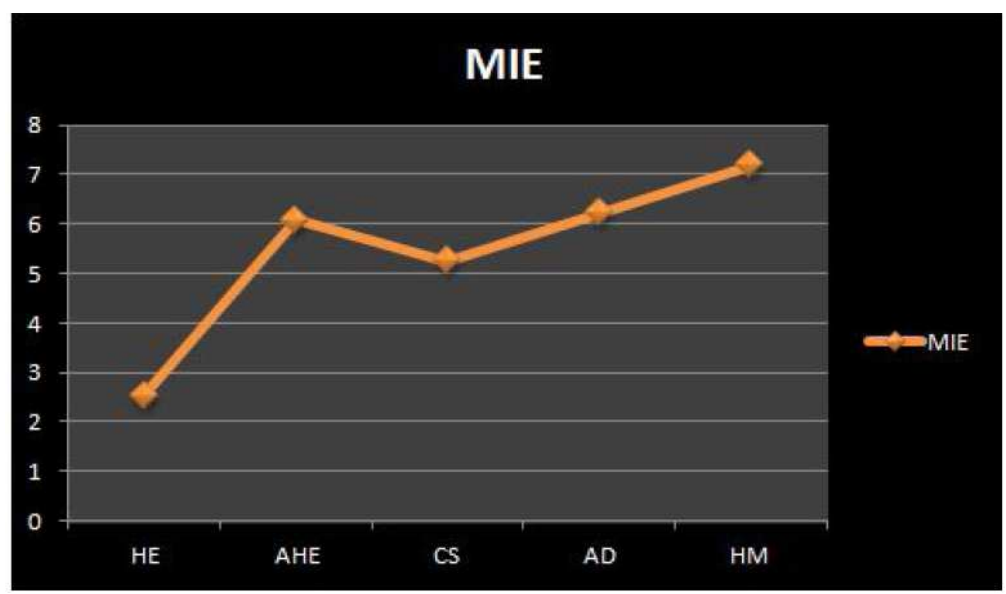

Fig. 8: MIE results for image enhancement algorithms

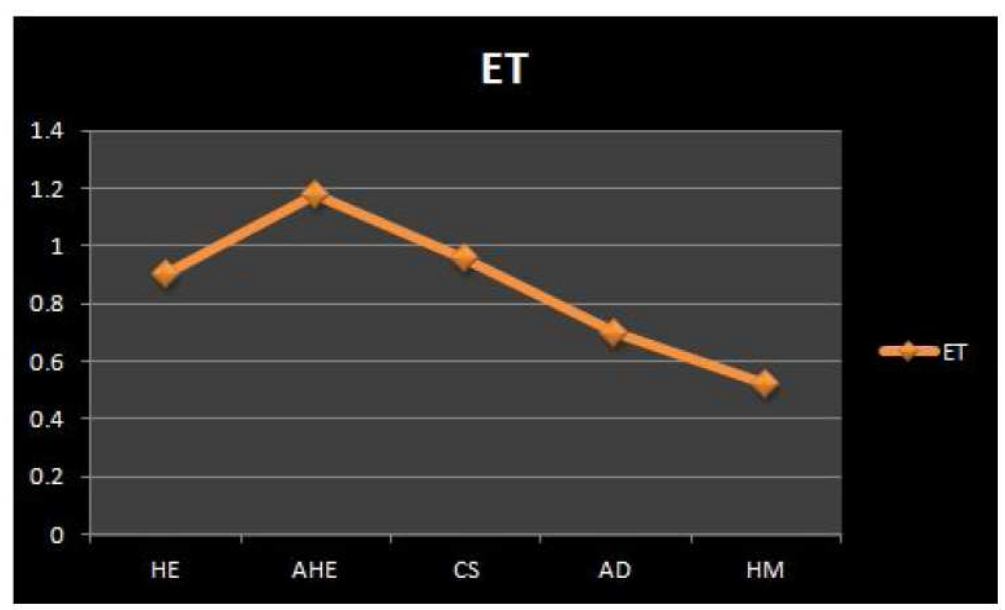

Fig. 9: ET results for image enhancement algorithms

The Fig. 7 shows the UQI for image enhancement algorithms. The PNA gives high value compares to other traditional methods.

Measurement of Image Enhancement (MIE): Let an image $x(n, m)$ be split into $k_{1} k_{2}$ blocks $W_{k, 1}(I, j)$ of sizes $1_{1}, l_{2}$ and let and $\{\varnothing\}$ be a given class of orthogonal transforms used for image enhancement with enhancement parameters (or, vector parameter) $\alpha$, $\beta$ and $\gamma$ to be found, then it is (Agaian et al., 1995)

$$
\text { MIE }=\frac{\max }{\varnothing \in\{\varnothing\}} X\left(\frac{1}{k_{1} k_{2}} \sum_{i=1}^{k=2} \sum_{i=2}^{k=1} 20 \log _{10} \frac{I_{\max ; k, 1}^{\mathrm{w}}}{I_{\min ; k, 1}^{\mathrm{w}}}\right)
$$

where, $I_{\text {min:k, }, 1}^{\mathrm{w}}$ and $I_{\text {max } k, 1,}^{\mathrm{w}}$ are respectively minimum and maximum of the image $x(m, n)$ inside the block $\mathrm{W}_{\mathrm{k}, \mathrm{l}}(\mathrm{I}$, j). The function $X$ is the sign function.
The Fig. 8 shows the MIE for image enhancement algorithms.

Evaluation Time (ET): Of a filter is defined as the time taken by a digital computing platform to execute the filtering algorithms. The execution time taken by a filter should be low for online and real-time image processing applications. Hence, a filter with lower ET is better than a filter having higher ET value when all other performance-measures are identical.The Fig. 9 shows the ET value for image enhancement algorithms.

\section{DISCUSSION}

The results are analysed based on Peak Signal to Noise Ratio (PSNR), Mean Square Error (MSE), Mean Absolute Error (MAE), Root Mean Square Error 


\section{J. Computer Sci., 8 (8): 1329-1337, 2012}

(RMSE), Universal Quality Index (UQI), Measure of Enhancement (or improvement) (MSE) (Agaian et al., 2007) and Elapsed Time (ET). The PSNR, UQI and MIE value must be high for a medical image, MSE, RMSE and ET must be low value for a better algorithm. The Fig. 4 shows the PSNR for image enhancement algorithms. The HM gives suitable results compare to traditional methods. The Fig. 5 shows the MSE for image enhancement algorithms. The HM gives low value compares to other traditional methods based on MSE and ET parametric values. The PNA gives high value compares to other traditional methods based on UQI and MIE.

\section{CONCLUSION}

This study describes the method to improve the image quality of DICOM image. The goal of the image enhancement technique is to improve a characteristic or quality of an image, such that the resulting image is better than the original image. One of the most common degradations in medical images are their poor contrast quality and noise. The DICOM image consists of speckle (multiplicative noise). While the image is enhanced, the multiplicative noise present in the images is also enhanced. To avoid this effect, the DICOM image is passed through a speckle filter. This suggests the use of contrast enhancement methods as an attempt to modify the intensity distribution of the image and to reduce the multiplicative noise. The result obtained proves the efficiency of the hybrid approach.

\section{REFERENCES}

Agaian, S., J. Astola, K. Egiazarian and P. Kuosmanen, 1995. Decompositional methods for stack filtering using Fibonacci p-codes. Signal Proces., 41: 101110. DOI: 10.1016/0165-1684(94)00093-F

Agaian, S.S., B. Silver and K.A. Panetta, 2007. Transform coefficient histogram-based image enhancement algorithms using contrast entropy. IEEE Tran. Proc., 16: 741-758. DOI: 10.1109/TIP.2006.888338

Ahmed, H.S.S. and M.J. Nordin, 2011. Improving diagnostic viewing of medical images using enhancement algorithms. J. Comput. Sci., 7: 18311838. DOI: $10.3844 /$ jcssp.2011.1831.1838

Al-Samaraie, M.F. and N.A.M.A. Saiyd, 2008. Medical colored image enhancement using wavelet transform followed by image sharpening. Ubiquitous Comput. Commun. J., 6: 1-8.

Gerig, G., O. Kubler, R. Kikinis and F.A. Jolesz, 2002. Nonlinear anisotropic filtering of MRI data. IEEE Trans. Med. Imaging, 11: 221-232. DOI: 10.1109/42.141646
Ismail, W.Z.W. and K.S. Sim, 2011. Contrast enhancement dynamic histogram equalization for medical image processing application. Int. J. Imaging Syst. Technol., 21: 280-289. DOI: 10.1002/ima.20295

Jagatheeswari, P., S.S. Kumar and M. Rajaram, 2009. Contrast enhancement for medical images based on histogram equalization followed by median filter. Proceedings of the 2nd International Conference on Man-Machine System, Oct. 11-13, Batu Ferringhi, Penang, Malaysia, pp: 2A41-2A44.

Kabir, H., A. Al-Wadud and O. Chae, 2010. Brightness preserving image contrast enhancement using weighted mixture of global and local transformation functions. Int. Arab J. Inform. Technol., 7: 403-410.

Kachouie, N.N., 2008. Anisotropic diffusion for medical image enhancement. Int. J. Image Proces. (IJIP), 3: 436-443.

Larrabide, I., A.A. Novotny, R.A. Feijoo and E. Taroco, 2003. A medical image enhancement algorithm based on topological derivative and anisotropic diffusion. Proceedings of the 26th Iberian LatinAmerican Congress on Computational Methods in Engineering CILAMCE, Oct. 19-21, Espirito Santo, pp: 1-14.

Lavania, K.K., Shivali and R. Kumar, 2011. A Comparative study of Image Enhancement using Histogram Approach. Int. J. Comput. Appli., 32: 1-6.

Mahmoud, T.A. and S. Marshal, 2008. Medical image enhancement using threshold decomposition driven adaptive morphological filter. Proceedings of the 16th European Signal Processing Conference, Aug. 25-29, Copyright by EURASIP, Switzerland, pp: 1-5.

Nakayama, K., W. Ohyama, T. Wakabayashi, F. Kimura and S. Tsoruoka et al., 2009. Speckle reduction of echocardiograms via wavelet shrinkage of ultrasonic RF signals. Int. Conf. Biomed. Eng., 23: 395-398. DOI: 10.1007/978-3540-92841-6_97

Perona, P. and J. Malik, 1990. Scale-space and edge detection using anisotropic diffusion. IEEE Trans. Patt. Anal. Machine Int., 12: 629-639. DOI: 10.1109/34.56205

Ramyashree, N., P. Pavithra, T.V. Shruthi and J. Majumdar, 2010. Enhancement of aerial and medical image using multi resolution pyramid. IJCCT, 1: 202-209.

Sapiro, G., A. Tannenbaum, Y.L. You and M. Kaveh, 1994. Experiments on geometric image enhancement. Proceedings of the IEEE International Conference on Image Processing, Nov. 13-16, IEEE Xplore Press, pp: 472-476. DOI: 10.1109/ICIP.1994.413615 
Vij, K. and Y. Singh, 2011. Comparison between different techniques of image enhancement. Int. J. VLSI Signal Proces. Appli., 1: 112-117.

Zhao, D., D. Jia, F. Han and J. Yang, 2010. A synchronization algorithm of mri denoising and contrast enhancement based on PM-CLAHE model. Int. J. Digital Content Technol. Appli., 4: 144-149. DOI: 10.4156/jdcta.vol4.issue6.17
Ziaei, A., H. Yeganeh, K. Faez and S. Sargolzaei, 2008. A novel approach for contrast enhancement in biomedical images based on histogram equalization. Proceedings of the International Conference on Bio-Medical Engineering and Informatics, May 27-30, IEEE Xplore Press, Tehran, pp: 855-858. DOI: 10.1109/BMEI.2008.300 